\title{
Carcinoid and hypocoagulation state in geriatric patients: a rare
}

\section{case}

\section{Carla Migliaccio*, Pamela Delmonaco, Eriberto Farinella, Francesco La Mura, Alessandro Spizzirri, Barbara Rossetti, Valerio Mecarelli, Roberto Cirocchi, Giammario Giustozzi and Francesco Sciannameo}

Address: Department of General and Emergency Surgery, S. Maria Hospital, Terni-University of Perugia, Italy

* Corresponding author

from XXI Annual Meeting of The Italian Society of Geriatric Surgery

Terni, Italy. 4-6 December 2008

Published: I April 2009

BMC Geriatrics 2009, 9(Suppl I):A100 doi:10.1 186/I47I-23I8-9-SI-A100

This abstract is available from: http://www.biomedcentral.com/|47I-23I8/9/SI/AI00

(C) 2009 Migliaccio et al; licensee BioMed Central Ltd.

\section{Background}

Recently, in literature, it is possible to find cases about the ulterior risk of serious haemorrhages associated to the selective serotonin reuptake inhibitors usage (SSRI) in geriatric patients. Neuroendocrine tumours are quite rare (one person out of 100,000), the most common localization is the small intestine and they represent $50 \%$ of the malignant tumours of small intestine.

\section{Materials and methods}

We observed a 79-year-old patient suffering from high blood pressure and Alzheimer, with an history of periodic abdominal pain from almost 2 months and $10 \mathrm{Kg}$ weight loss in the last month. In suspicious of a carcinoid, the patient underwent scintigraphy with In-111 showing the presence of two abnormal focal buildup of the trace compatible with the presence of a productive tissue with somatostatin receptors. Lab exams were normal, except for the serum Chromogranin A $(42.0 \mathrm{U} / \mathrm{l})$, the $5 \mathrm{OH}$ indole acetic acid $(11.0 \mathrm{mg} / 24 \mathrm{~h})$ and the urinary VanillylMandelic acid $(7.0 \mathrm{mg} / 24 \mathrm{~h})$. Therefore the patient underwent surgery. The patient had a regular course until the third post-operative day when an acute anemia appeared (GR 2460000, Hb $7.0 \mathrm{~g} / \mathrm{dl}, \mathrm{HCT} 22.3 \%$ ). Therefore we performed an emergency intervention that showed the presence of diffuse bleeding from the parietal and visceral peritoneum with haemorragic filling of the transverse mesocolon.

\section{Results}

The association between carcinoid and hypocoagulative state is rare and their connection is not yet clear.

Hemocoagulative alterations can be often found in a tumor. The aberrant production of procoagulant and hematopoietic growth factors, fibrinolytic and proteolytic factors is responsible for several paraneoplastic syndromes. The increased risk of serious haemorrhages in association with SSRI is a consequence of the inhibition of the platelet aggregation caused by SSRI through serotonin depletion in platelets. In 1999, this article's authors had already demonstrated that SSRI can increase the risk of bleeding of the upper gastrointestinal tract. This risk is increased especially with concomitant therapy with aspirin or nonsteroidal anti-inflammatory agents or by stress factors (major surgery, sepsis, shock, etc) in geriatric patients.

\section{Discussion}

The absence of a precise location of postoperative bleeding can be explained by the hypocoagulative state of the patient due to different factors: coagulopathy by paraneoplastic syndrome, assumption of selective serotonin reuptake inhibitors, geriatric patient and operative stress. 